\title{
Physicochemical, structural and induced ferromagnetic properties of Co-In-codoped CdO synthesised via Cd chloride: significant effect of post-treatment hydrogen
}

\author{
A A DAKHEL* and W E ALNASER \\ Department of Physics, College of Science, University of Bahrain, P.O. Box 32038, Bahrain, Kingdom of Bahrain
}

MS received 26 February 2016; accepted 4 May 2016

\begin{abstract}
Nanoparticle solid solution powders of cadmium oxide doped with different concentrations of cobalt and indium ions were synthesised by solvothermal method through $\mathrm{CdCl}_{2} \cdot \mathrm{H}_{2} \mathrm{O}$ precursor route. The objective of the present work is to study and develop conditions necessary to create stable room-temperature ferromagnets (RTFMs) in transparent conducting oxide (TCO) $\mathrm{CdO}$ for applications in the field of dilute magnetic semiconductors (DMSs). To achieve this aim, cobalt $\left(\mathrm{Co}^{2+}\right)$ dopant ions were used as a source of stable FM, while $\mathrm{In}^{3+}$ dopant ions $^{3}$ supply free electrons that enhance the electronic medium of interaction. The electronic medium in the host CdO lattice, which carries the long-range spin-spin (S.S) exchange interaction between localised $\mathrm{Co}^{2+}(3 \mathrm{~d})$ spins of dopant ions, was further developed by annealing in hydrogen gas (hydrogenation). The crystalline structure of the powder samples was investigated by the X-ray diffraction (XRD) method. The optical absorption properties were studied by diffuse reflection spectroscopy (DRS). Magnetic measurements reveal that the Co-In-codoped CdO powder has FM properties superimposed on paramagnetic (PM) behaviour. However, annealing in hydrogen atmosphere strongly boosts the created FM so that the saturation magnetisation increases $\sim 90$ times. Physical explanations and discussion are given in the article. Thus, it is proved that the magnetic properties could be tailored to TCO CdO by Co-doping and post-treatment under $\mathrm{H}_{2}$ atmosphere.
\end{abstract}

Keywords. Co-In-codoped CdO; room-temperature ferromagnetism; hydrogen treatment.

\section{Introduction}

Transparent conducting oxides (TCOs) are a group of metallic oxides like $\mathrm{ZnO}, \mathrm{CdO}, \mathrm{In}_{2} \mathrm{O}_{3}, \mathrm{SnO}_{2}$ and $\mathrm{Ga}_{2} \mathrm{O}_{3}$ that have many industrial applications, especially in optoelectronics [1]. Their optical and electrical properties could be controlled by means of their natural structural point defects. One of the methods by which we can control their structural defects is doping with foreign metallic ions. It was found experimentally that doping of TCOs with some metallic ions could increase the concentration of carriers and/or improve the conductivity [2]. Besides, the doping process could generate extra exotic properties in TCOs, such as magnetic, optical or mechanical properties, that could widen the fields of their applications. The present study focuses on doping of $\mathrm{CdO}$ for developing conditions necessary to create stable roomtemperature ferromagnets (RT-FMs) for applications in the field of dilute magnetic semiconductors (DMSs). To achieve this purpose, it is essential to dope $\mathrm{CdO}$ with some transition or rare-earth ions, which have intrinsic magnetic moments. Furthermore, it is required to think about developing the electronic medium in the host crystal $(\mathrm{CdO})$ that is necessary to hold the spin-spin $(\boldsymbol{S} . S)$ exchange long-range interaction between localised spins of the dopant ions.

\footnotetext{
*Author for correspondence (adakhil@uob.edu.bh)
}

Indeed, more theoretical and experimental works are required to clarify and explain the mechanism by which RTFM could be created in DMS materials with tiny doping level or even, sometimes, without doping. Some researchers attribute the creation of RT-FM in undoped oxides to their intrinsic point defects, such as oxygen $(\mathrm{O})$ vacancies [3-5]. However, the role of O-vacancies in creating/boosting RT-FM is still under study.

In the present work, dopants $\mathrm{Co}^{2+}$ magnetic ions are used as a source of stable FM in CdO. It is known that the magnetic moment of $\mathrm{Co}^{2+}$ ion is 4.2-4.8 $\mu_{\mathrm{B}}$ (where $\mu_{\mathrm{B}}$ is Bohr magneton) as measured experimentally in some tetrahedral complexes [6]. In addition, some facts related to the doping process should be mentioned: the VI-coordination ionic radius of $\mathrm{Cd}^{2+}(0.095 \mathrm{~nm})$ is close to that of $\mathrm{Co}^{2+}(0.0745$ $\mathrm{nm}$ ) [7] and the crystalline structures of $\mathrm{CdO}$ and $\mathrm{CoO}$ are identical (rock-salt Fm-3m). Therefore, high solubility of $\mathrm{Co}^{2+}$ ions in $\mathrm{CdO}$ is expected, forming substitutional solid solution (SSS). Furthermore, the $\mathrm{In}^{3+}$ ionic radius $(0.08 \mathrm{~nm}$, coordination VI) [7] is less than that of $\mathrm{Cd}^{2+}$, which permits $\mathrm{In}^{3+}$ ions to occupy the positions of $\mathrm{Cd}^{2+}$ ions in $\mathrm{CdO}$ crystal without disturbing the crystalline structure.

Several usual chemical and physical methods are used to synthesise metal-doped $\mathrm{CdO}$ powder; however, in the present work, pure and doped $\mathrm{CdO}$ powders were synthesised by means of thermal decomposition of $\mathrm{Cd}$ chloride 
$\left[\mathrm{CdCl}_{2} \cdot \mathrm{H}_{2} \mathrm{O}\right]$. Moreover, the synthesis procedure involved using dilute $\mathrm{HCl}$ acid.

Cobalt ions were used as a dopant to many oxides, like $\mathrm{SnO}_{2}$ [8] or $\mathrm{In}_{2} \mathrm{O}_{3}$ [9] in order to study their magnetic properties, where paramagnets with weak antiferromagnetism (AFM) interactions were observed. Paramagnetic (PM) behaviour was observed in Co-doped $\mathrm{CdO}$ powder prepared by thermal decomposition of organic complexes [10]. Moreover, weak AFM superimposed on major PM properties was observed at low temperatures $(5 \mathrm{~K})$ in Co-doped $\mathrm{CdO}$ system [11]. However, FM behaviour was observed in Codoped $\mathrm{ZnO}$ powder, which was explained by the presence of oxygen vacancies and zinc interstitials [12]. Thus the created magnetic behaviour qualitatively depends on the kind of dopants and host, and the details of the used procedure of synthesis.

\section{Experimental}

Nanoparticle solid solution powders of Co-In-codoped CdO have been synthesised by solvothermal method through $\mathrm{CdCl}_{2} \cdot \mathrm{H}_{2} \mathrm{O}$ precursor route. Pure $\mathrm{Cd}$, In and Co fine powders of analytical grade (supplied from Sigma-Aldrich products) and $\mathrm{HCl}$ were used as starting materials in order to synthesise pure and Co-In-codoped cadmium chloride $\left(\mathrm{CdCl}_{2} \cdot \mathrm{H}_{2} \mathrm{O}\right)$ precursor powder. The procedure of synthesise is quite simple. A mixture of controlled amounts of $\mathrm{Cd}$, In and $\mathrm{Co}$ fine powders was dissolved in dilute $(\sim 3 \%) \mathrm{HCl}$ acid under continuous mild magnetic stirring at RT for $\sim 24 \mathrm{~h}$, until the solution color changed to that of fat milk. Finally, the suspended white precipitate was collected by slow evaporation at $80^{\circ} \mathrm{C}$. The produced precipitate powder was flash calcined in a closed oven at $500^{\circ} \mathrm{C}$ for $1 \mathrm{~h}$ followed by natural cooling to room temperature. The yielded white precursor powder was tested by the X-ray diffraction (XRD) method, which established that it had just the $\mathrm{CdCl}_{2} \cdot \mathrm{H}_{2} \mathrm{O}$ structure. Next, the synthesised precursor powder was thermally decomposed by calcination at $\sim 800^{\circ} \mathrm{C}$ for $\sim 2 \mathrm{~h}$ in order to obtain the final product of $\mathrm{CdO}$ crystalline structure product. It must be mentioned here that by thermochemistry analysis it was found [13] that during the decomposition of $\mathrm{CdCl}_{2} \cdot \mathrm{H}_{2} \mathrm{O}$ to $\mathrm{CdO}$, it lost $61 \%$ of its weight by volatilisation.

Two concentrations of cobalt dopant were prepared. The ratios $\mathrm{Co} / \mathrm{Cd}$ were 10 at\% for the $\mathrm{CdO}: \mathrm{Co}-\mathrm{In}$ (S1) sample and 30 at\% for sample CdO:Co-In (S2), while only one $\mathrm{In} / \mathrm{Cd}$ concentration ratio of 3 at\% was used in both samples. Finally, some amount of the final as-synthesised $\mathrm{CdO}$ product powder was post-annealed in hydrogen atmosphere at $350^{\circ} \mathrm{C}$ for $30 \mathrm{~min}$.

The elemental analysis of the samples was performed by the X-ray fluorescence (XRF) method. The structural analysis of the samples was carried out using a Rigaku Ultima VI $\theta-2 \theta$ X-ray diffractometer with $\mathrm{Cu} \mathrm{K}_{\alpha}$ rays. The optical properties of the prepared powder samples were studied by diffuse reflectance spectroscopy (DRS). The magnetic properties were measured with a Micro-Mag Model 3900.

\section{Results and discussion}

\subsection{Structural analysis}

The XRF spectra of the synthesised final host CdO samples $\mathrm{S} 1$ and $\mathrm{S} 2$ are depicted in figure 1. The spectra disclose the elemental contents and, hence, the purity of the synthesised powders. They show Cd L-spectrum (3.13-3.53 keV) and Co $\mathrm{K}_{\alpha}$ and $\mathrm{K}_{\beta}$ signals (6.93 and $7.65 \mathrm{keV}$, respectively), and the

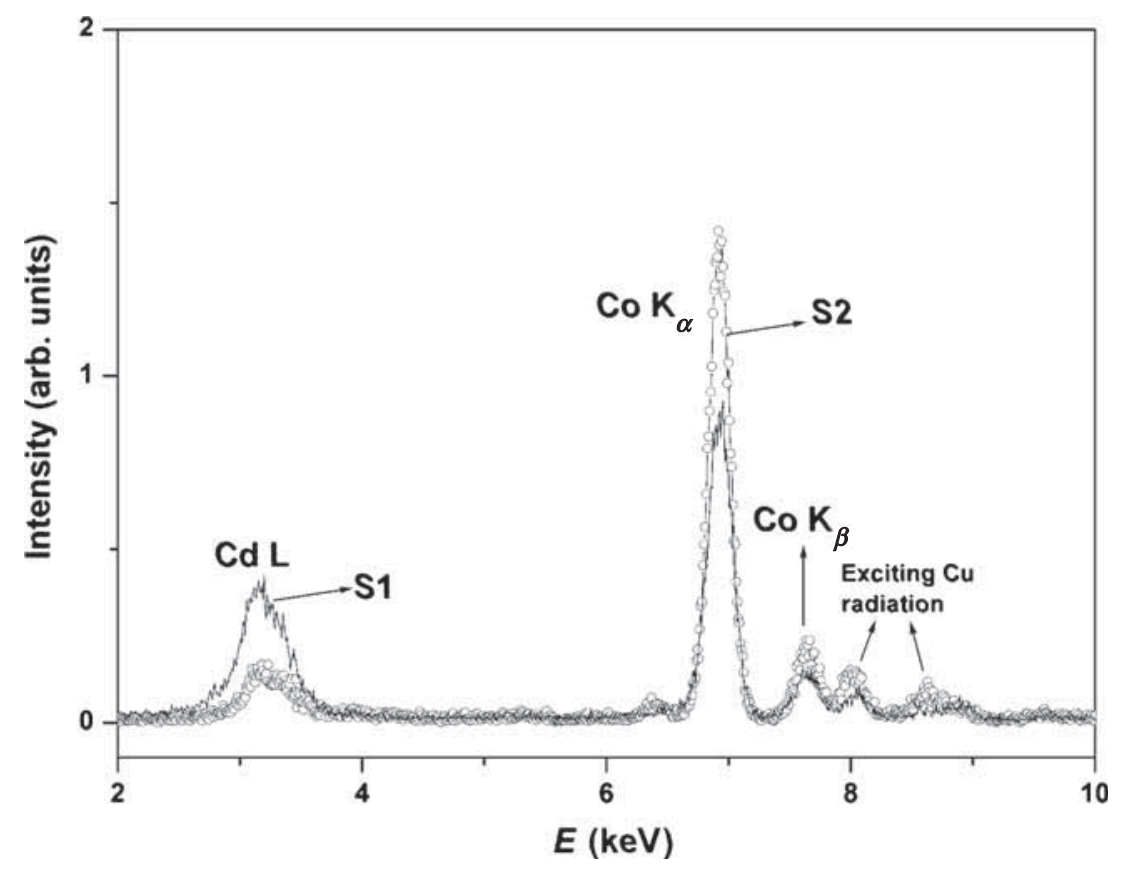

Figure 1. XRF spectra of the prepared powder $\mathrm{S} 1$ and $\mathrm{S} 2$ samples. 
exciting $\mathrm{Cu} \mathrm{K}_{\alpha}$ and $\mathrm{K}_{\beta}$ signals (8.04 and $8.90 \mathrm{keV}$, respectively). The In signal ( $3.28 \mathrm{keV}$ ) completely overlapped with the Cd L-spectrum due to the small content of In. No additional XRF signals were detected, indicating to the elemental content and purity of the prepared samples.

Figure 2 shows the XRD patterns of the precursor powders. They were indexed according to the crystalline structure of cadmium chloride hydrate $\left(\mathrm{CdCl}_{2} \cdot \mathrm{H}_{2} \mathrm{O}\right)$, which is principally formed by the well-known chemical reaction $\mathrm{CdO}+2 \mathrm{HCl} \rightarrow \mathrm{CdCl}_{2}+\mathrm{H}_{2} \mathrm{O}$. The crystalline structure of $\mathrm{CdCl}_{2} \cdot \mathrm{H}_{2} \mathrm{O}$ is known to be orthorhombic Pnam (62) with parameters $a=0.92610 \mathrm{~nm}, b=1.17300 \mathrm{~nm}, c=0.37940$ $\mathrm{nm}$ and $V_{\text {cell }}=0.412 .148 \mathrm{~nm}^{3}$ [14]. In the present work, the refinement parameters by the Rietveld method for the precursor powders of undoped and Co-In-codoped $\mathrm{CdO}$ powders are given in table 1 . The results prove the total incorporation of $\mathrm{In}^{3+}$ and $\mathrm{Co}^{2+}$ ions in the lattice of the synthesised solid precursors. Also, the XRD data reveal that the prepared $\mathrm{CdCl}_{2} \cdot \mathrm{H}_{2} \mathrm{O}$ precursors are nanocrystallite powders.

The XRD of final synthesised powders is shown in figure 3. The patterns reveal that the synthesised powders are polycrystalline $\mathrm{CdO}$ in cubic structure. The absence of diffraction peaks arising from pure or related $\mathrm{Co} / \mathrm{In}$ phases refers to the incorporation of $\mathrm{Co}^{2+} / \mathrm{In}^{3+}$ ions in the lattice of host $\mathrm{CdO}$, forming $\mathrm{S} 1$ and $\mathrm{S} 2$ solid solutions. The results of the structural analysis: lattice parameter (calculated by Rietveld crystal refinement), crystallite size (CS) and strain (calculated by the Halder-Wagner method), are listed in table 2.

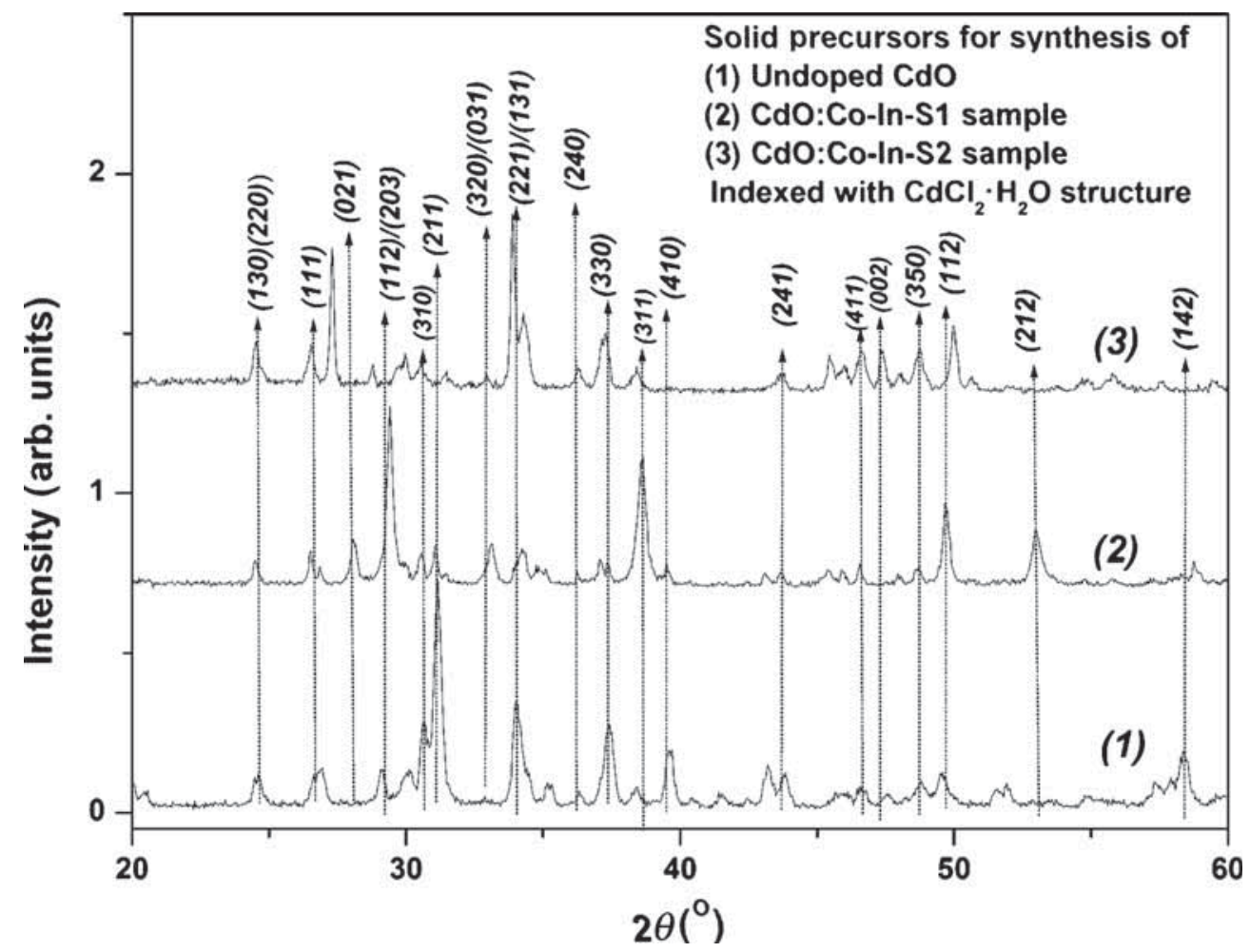

Figure 2. XRD patterns of the precursor powders for syntheses of undoped CdO, S1 and S2 samples.

Table 1. Structural analysis: crystallite size (CS), micro-strain, lattice parameters $\left(a, b\right.$ and $\left.c, V_{\text {cell }}\right)$ and Rietveld refinement quality parameters for precursor powders of synthesis of $\mathrm{CdO}, \mathrm{S} 1$ and $\mathrm{S} 2$ samples.

\begin{tabular}{|c|c|c|c|c|c|c|c|c|c|}
\hline \multirow[b]{2}{*}{ Sample } & \multirow[b]{2}{*}{$\mathrm{CS}(\mathrm{nm})$} & \multirow[b]{2}{*}{ Strain $(\%)$} & \multirow{2}{*}{$\begin{array}{c}\text { Lattice } \\
\text { parameters }(\AA)\end{array}$} & \multicolumn{6}{|c|}{ Refinement factors } \\
\hline & & & & $V_{\text {cell }}\left(\AA^{3}\right)$ & $R_{\mathrm{wp}}(\%)$ & $R_{\mathrm{p}}(\%)$ & $R_{\mathrm{e}}(\%)$ & $S$ & $\chi^{2}$ \\
\hline Precursor of undoped $\mathrm{CdO}$ & 20.7 & 0.15 & $\begin{array}{l}a=9.253 \\
b=11.735 \\
c=3.790\end{array}$ & 411.5 & 39.37 & 30.95 & 13.23 & 2.9764 & 8.8592 \\
\hline Precursor of CdO:Co-In-S1 & 23.7 & 0.18 & $\begin{array}{l}a=9.259 \\
b=11.729 \\
c=3.7916\end{array}$ & 411.7 & 25.08 & 18.57 & 16.5 & 1.5186 & 2.3061 \\
\hline Precursor of $\mathrm{CdO}: \mathrm{Co}-\mathrm{In}-\mathrm{S} 2$ & 21.4 & 0.10 & $\begin{array}{l}a=9.251 \\
b=11.727 \\
c=3.793\end{array}$ & 411.5 & 33.78 & 24.58 & 14.76 & 2.2888 & 5.2388 \\
\hline
\end{tabular}




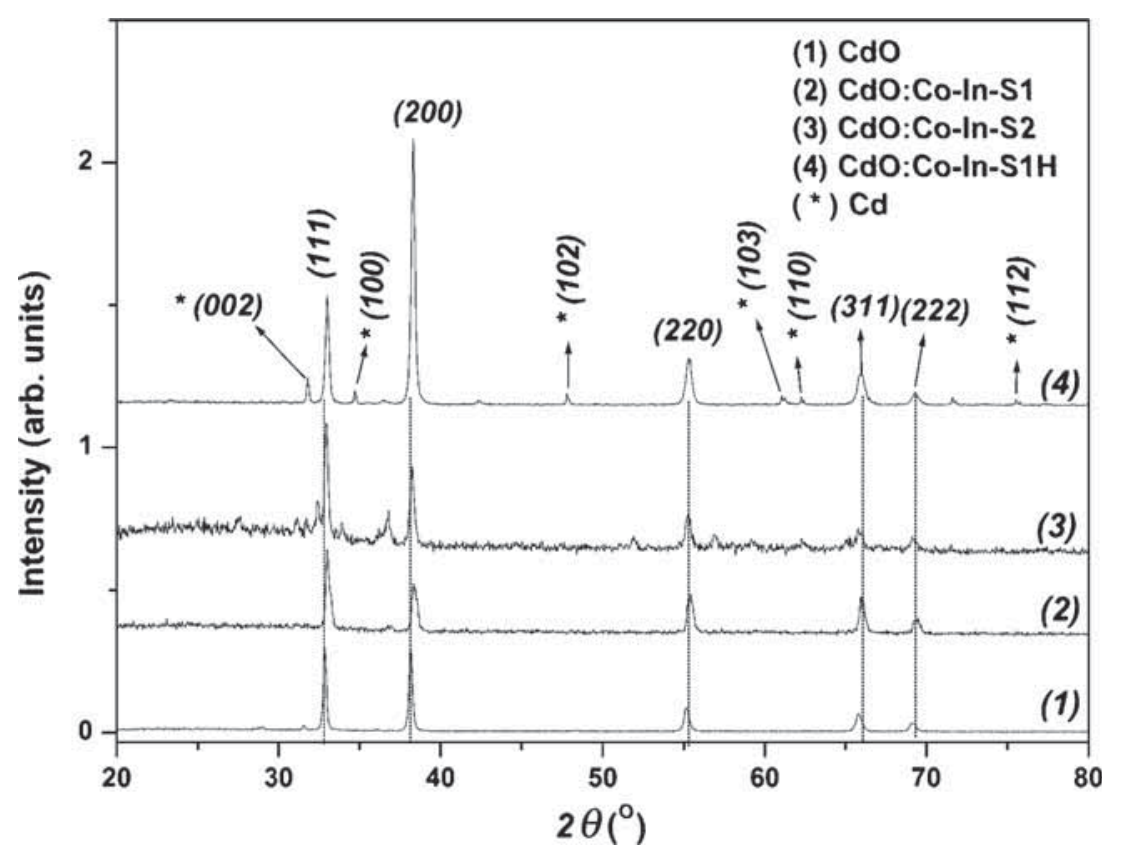

Figure 3. XRD patterns of samples $\mathrm{CdO}, \mathrm{S} 1, \mathrm{~S} 2$ and $\mathrm{S} 1-\mathrm{H}$ powders.

Table 2. Structural analysis: crystallite size (CS), the structural micro-strain, lattice parameter $(a)$, Rietveld refinement quality parameters and optical bandgap $\left(E_{\mathrm{g}}(\mathrm{eV})\right)$.

\begin{tabular}{|c|c|c|c|c|c|c|c|c|c|}
\hline \multirow[b]{2}{*}{ Sample } & \multirow[b]{2}{*}{$\mathrm{CS}(\mathrm{nm})$} & \multirow[b]{2}{*}{ Strain $(\%)$} & \multirow{2}{*}{$\begin{array}{c}\text { Lattice } \\
\text { parameter } a(\AA)\end{array}$} & \multicolumn{6}{|c|}{ Refinement factors } \\
\hline & & & & $R_{\mathrm{wp}}(\%)$ & $R_{\mathrm{p}}(\%)$ & $R_{\mathrm{e}}(\%)$ & $S$ & $\chi^{2}$ & $\overline{E_{\mathrm{g}}}$ \\
\hline $\mathrm{CdO}$ & 73.2 & 0.26 & 4.7044 & 16.98 & 13.65 & 11.4 & 1.487 & 2.211 & 2.1 \\
\hline $\mathrm{CdO}: \mathrm{Co}-\mathrm{In}-\mathrm{S} 1$ & 25.9 & 0.21 & 4.6927 & 17.37 & 13.73 & 15.15 & 1.144 & 1.310 & 2.05 \\
\hline $\mathrm{CdO}: \mathrm{Co}-\mathrm{In}-\mathrm{S} 1 \mathrm{H}$ & 66.7 & 0.32 & 4.6961 & 36.81 & 29.92 & 11.96 & 3.072 & 9.437 & 2.90 \\
\hline $\mathrm{Cd}(16.7 \%)$ & 42.8 & 0.09 & $\begin{aligned} a & =2.9802, \\
c & =5.6190\end{aligned}$ & 25.08 & 21.16 & 211.91 & 2.101 & 4.417 & \\
\hline $\mathrm{CdO}: \mathrm{Co}-\mathrm{In}-\mathrm{S} 2$ & 40.6 & 0.23 & 4.702 & 16.96 & 12.87 & 11.81 & 1.434 & 2.057 & 1.90 \\
\hline
\end{tabular}

The lattice constant of undoped $\mathrm{CdO}$ powder was found to be close to the value given in JCPDS Card No. 05-0640 (Fm$3 \mathrm{~m}, a=0.4695 \mathrm{~nm}$ ) (JCPDS File No. 05-0640). However, the volume of the unit cell $\left(V_{\text {cell }}=\mathrm{a}^{3}\right)$ of host $\mathrm{CdO}$ slightly reduced, comparing with undoped $\mathrm{CdO}$, due to the doping by $\mathrm{Co}^{2+} / \mathrm{In}^{3+}$ ions of slightly smaller ionic radius than that of $\mathrm{Cd}^{2+}$. The monophase of S2 with high Co doping level shows that cobalt ions have high solubility in $\mathrm{CdO}$. The substitution of $\mathrm{Cd}^{2+}$ ions by $\mathrm{Co}^{2+}$ ions would not strongly disturb the crystalline structure and did not disturb the charge neutrality of the unit cell. Therefore, the substitution for $\mathrm{Cd}^{2+}$ ions by $\mathrm{Co}^{2+}$ ions is the most likely to occur, forming SSS in addition to the possible occupation by $\mathrm{Co}^{2+}$ of some interstitial positions in $\mathrm{CdO}$ structure. However, the occupation of $\mathrm{Cd}^{2+}$ positions by $\mathrm{In}^{3+}$ ions disturbs the charge neutrality of the unit cell, which would be settled by redistribution of ions in the unit cell. The XRD pattern of hydrogenated S1 sample (i.e., S1H) is shown in figure 3, which reveals two major structural modifications. First, extra weak peaks appear in the XRD pattern, which are attributed to the formation of $\mathrm{Cd}$ metal nanograins. The XRD results for the liberated $\mathrm{Cd}$ grains given in table 2 confirm that they are of nano-size with hexagonal structure and parameters $a=0.29802 \mathrm{~nm}$ and $c=$ $0.56190 \mathrm{~nm}$, which are close to the previously known HCP $\left(\mathrm{P}_{3} / \mathrm{mmc}\right)$ structure with lattice parameters $a=0.29794 \mathrm{~nm}$ and $c=0.56186 \mathrm{~nm}$ [15]. The liberated $\mathrm{Cd}$ ions are due to the interaction between hydrogen and structural oxygen ions that leads to their removal from the host $\mathrm{CdO}$ crystalline structure, forming oxygen vacancies $\left(\mathrm{V}_{\mathrm{O}}\right)$. The removal of structural oxygen ions disturbs the electro-neutrality of the host $\mathrm{CdO}$ unit cell that would be settled by liberation of $\mathrm{Cd}$ ions outside the host $\mathrm{CdO}$ lattice.

Due to the liberation and expelling of $\mathrm{Cd}$ ions, the lattice imperfection/strain increased by about 1.5 times (table 2). Furthermore, the highest peak intensity position changed from (111) in S1 to (200) in S1H (figure 3). Another observation is that the CS considerably grew by about 2.5 times (table 2). This might be explained by the movement of 
the ions by hydrogen impacts that increases the agglomerations on crystallites or gradual removal of the crystallite boundaries. The reduction of oxygen content increases the unit cell volume $\left(V_{\text {cell }}\right)$, which is clearly observed in data of table 2. Such observation was also previously mentioned in Refs. [16,17].

To completely understand the effect of hydrogenation, it should be mentioned that $\mathrm{H}_{2}$ molecules might be dissociated into $\mathrm{H}$ atoms on the surface of nanocrystallites and nanograins due to the presence of Co ions, playing a role of catalyst since the transition metals (TMs) are well known to possess high catalytic effect for $\mathrm{H}_{2}$ molecules dissociation $[18,19]$. Later, the $\mathrm{H}$ atoms will be adsorbed at the surface of nanoparticles and diffuse into the bulk through interstitial sites or vacancies within $\mathrm{CdO}$ crystal lattice followed by interaction with structural oxygen, forming oxygen vacancies and thus boosting the magnetic properties [20]. Therefore, it should be expected that sample $\mathrm{S} 1 \mathrm{H}$ must have stronger FM properties than those of S1. It should be mentioned that this effect did not happen with pure undoped $\mathrm{CdO}$; it must need a metallic catalyst like $\mathrm{TM}$ ions to be involved. Therefore, as a general conclusion, hydrogenation of TM-doped $\mathrm{CdO}$ would produce a nanocomposite crystal consisting of $\mathrm{Cd}$ nanoparticles immersed in TM-doped $\mathrm{CdO}$ crystalline solid medium.

\subsection{Optical properties}

The optical properties of the synthesised powders were studied by the DRS method. The spectral diffuse reflectance $R(\lambda)$ was measured in the range $250-900 \mathrm{~nm}$ relative to the standard reference reflectance $\left(R_{\mathrm{BaSO}_{4}}\right)$ from a $\mathrm{BaSO}_{4}$ powder supplied by Schemadzu, i.e., $R(\lambda)=R_{\text {sample }} / R_{\mathrm{BaSO}_{4}}$, and the results are shown in the inset of figure 4 . These $\mathrm{R}$-spectra are usually used to estimate the bandgap of the powders using the Kubelka-Munk (K-M) equation [21]

$$
K / S=F(R)=(1-R)^{2} / 2 R,
$$

where $F(R)$ is called as the K-M function, $S$ is the scattering coefficient and $K$ is called as the $\mathrm{K}-\mathrm{M}$ absorption coefficient, where $K=2 \alpha$ and $\alpha$ is the absorption coefficient. Considering $S$ as a constant with respect to the wavelength $(\lambda)$, it is possible to use $F(R)$ in the well-known Tauc plot technique in order to estimate the direct $E_{\mathrm{g}}$, i.e., the extrapolation of the linear portion of $[F(R) \times E]^{2} v s$. $E$ plot, as shown in figure 4 . The obtained direct bandgaps are given in table 2. The bandgap found for undoped synthesised $\mathrm{CdO}$ nanopowder is consistent with the known range $(2.1-2.6 \mathrm{eV})$ for undoped $\mathrm{CdO}$ films [1]. However, with $\mathrm{Co} / \mathrm{In}$ incorporation, the bandgap is red-shifted. This result refers to the doping of $\mathrm{Co}^{2+} / \mathrm{In}^{3+}$ ions in $\mathrm{CdO}$ crystalline structure, which creates an impurity band at the tail of conduction band. The impurity band becomes wider with increasing Co doping level, which means larger decrease in $E_{\mathrm{g}}$, as given in table 2. This result is in agreement with Ref. [22]. Moreover, the hydrogenation blue-shifts the bandgap (table 2). Increase of bandgap $\left(E_{\mathrm{g}}\right)$ with hydrogenation could be explained by the Moss-Burstein effect [23]; i.e., $E_{\mathrm{g}}$ increases with increasing concentration of free carriers $\left(n_{\mathrm{el}}\right)$. Thus, the hydrogenation of semiconducting oxide $\mathrm{CdO}$ at relatively low temperature $\left(350^{\circ} \mathrm{C}\right)$ and for a short time period $(30 \mathrm{~min})$ is beneficial for generation of more free electrons, which might improve the electronic crystalline medium for boosting the spin-spin $(\boldsymbol{S} . S)$ interaction.

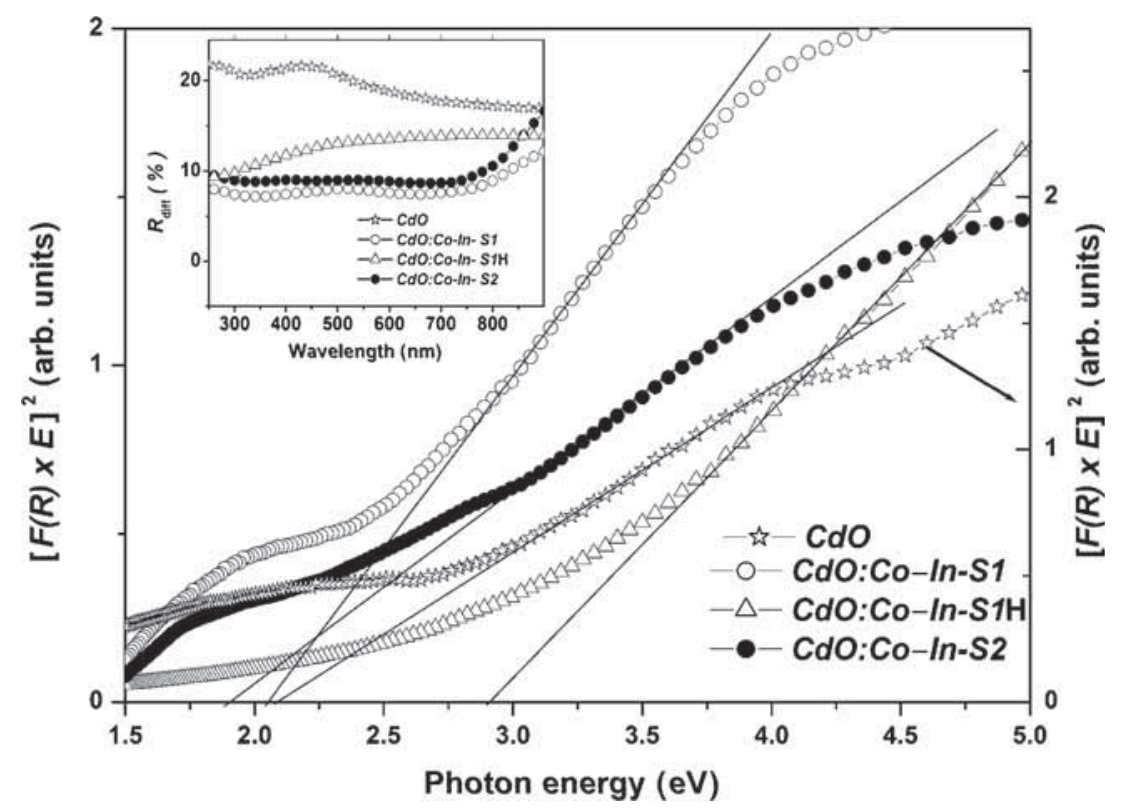

Figure 4. Tauc plot for the samples $\mathrm{CdO}, \mathrm{S} 1$ and $\mathrm{S} 1-\mathrm{H}$. The inset shows spectral diffuse reflectance for $\mathrm{CdO}, \mathrm{S} 1$ and $\mathrm{S} 1-\mathrm{H}$ powders. 


\subsection{Magnetic properties}

It is known that undoped, bulk and stoichiometric $\mathrm{CdO}$ powder has diamagnetic (DM) properties at RT [24]. However, it was observed that structural point defects like vacancies could create in $\mathrm{CdO}$ a very weak FM component superimposed on the main DM characteristics (i.e., DM+FM) [25]. However, for practical applications in DMS, it is necessary to create stable and considerable FM properties from spin-spin (S.S) exchange interaction between dopant ions. However, the realisation of $\boldsymbol{S} . \boldsymbol{S}$ exchange interaction depends on the interionic exchange distances $(R)$ and the properties of electronic medium of host crystal $(\mathrm{CdO})$ through which the $\boldsymbol{S} . \boldsymbol{S}$ interaction takes place. Generally, it was established that the $S . S$ exchange interaction requires $R \leq 1.5 a$, where $a$ is the lattice parameter of host medium [26]. $R$ could be estimated as radius of a sphere around each $\mathrm{Co}^{2+}$ ion. For a uniform distribution of $\mathrm{Co}^{2+}$ dopants within the host $\mathrm{CdO}$, the value of $R$ can be estimated by the relation $n_{\text {Co }} V=1$, where $n_{\text {Co }}$ is the dopant $\mathrm{Co}^{2+}$ concentration and $V=(4 / 3) \pi R^{3}$. Thus, the relative ionic concentration of dopant $\mathrm{Co}^{2+}$ to host $\mathrm{Cd}^{2+}$ should obey the relation $\left(n_{\mathrm{Co}} / n_{\mathrm{Cd}}\right) \geq 1.8 \%$ in order to switch on the $S . S$ exchange interaction. This condition is followed in the present work, which means that FM interaction should be present for a suitable electronic interaction medium, which can be controlled by the experimental details and conditions.

In the present work, FM properties were generated in $\mathrm{CdO}$ nanocrystallite powder synthesised from $\mathrm{Cd}$ chloride $\left(\mathrm{CdCl}_{2} \cdot \mathrm{H}_{2} \mathrm{O}\right)$ by means of $\mathrm{Co} / \mathrm{In}$-codoping (sample $\mathrm{S} 1$ ), as shown in figure 5. The FM parameters: coercive force $\left(H_{\mathrm{c}}\right)$, remanence $\left(M_{\mathrm{r}}\right)$ and saturation magnetisation $\left(M_{\mathrm{S}}\right)$ of $\mathrm{S} 1$, are given in table 3 , where the power product $U_{\mathrm{m}}=H_{\mathrm{c}} M_{\mathrm{r}}$ was introduced as a measure for FM figure of merit [27]. The $U_{\mathrm{m}}$ value for sample S1 is small, indicating weakly generated FM properties.

From basic principles, it is possible to boost the created FM properties in the sample $\mathrm{S} 1$ either by enhancing the strength of the spin-spin (S.S) interaction between the dopant $\mathrm{Co}^{2+}$ ions or by increasing the concentration of spins (S) participating in mutual S.S interaction, i.e., by increasing the Co-doping level. The first approach could be realised by enhancing the internal crystalline electronic medium S.S interaction through hydrogenation method. In the present work we would like to compare and discuss these two approaches for the enhancement of the created FM.

Figure 5 and table 3 present the experimental magnetic results for samples $\mathrm{S} 1, \mathrm{~S} 2$ and $\mathrm{S} 1 \mathrm{H}$. Data show that the hydrogenation of sample S1 strongly boosts its magnetic

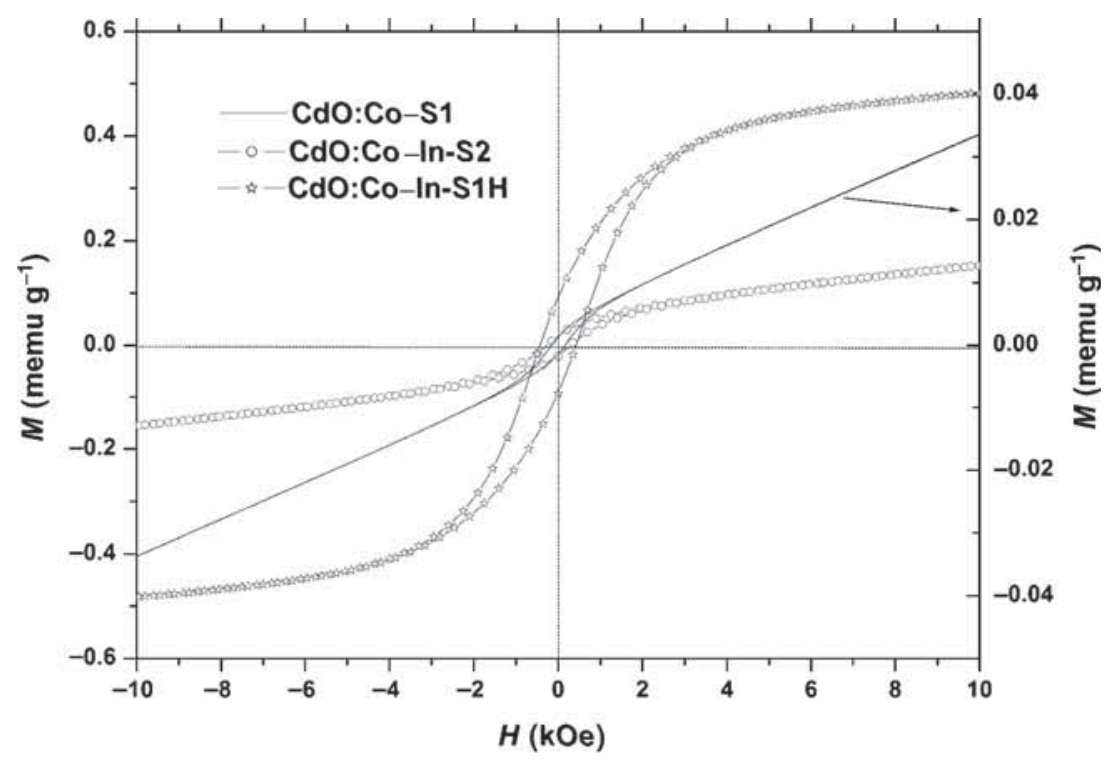

Figure 5. Experimental results of $M-H$ dependence of the samples S1, S2 and S1-H.

Table 3. Obtained values for the coercivity $\left(H_{\mathrm{c}}\right)$, remanence $\left(M_{\mathrm{r}}\right)$, saturation magnetisation $\left(M_{\mathrm{s}}\right)$ and energy product $\left(U_{\mathrm{m}}=H_{\mathrm{c}} M_{\mathrm{r}}\right)$ for the samples.

\begin{tabular}{lcccc}
\hline Sample & $H_{\mathrm{c}}(\mathrm{Oe})$ & $M_{\mathrm{r}}\left(\mathrm{memu} \mathrm{g}^{-1}\right)$ & $M_{\mathrm{s}}\left(\mathrm{memu} \mathrm{g}^{-1}\right)$ & $U_{\mathrm{m}}=H_{\mathrm{c}} M_{\mathrm{r}}(\mathrm{erg})$ \\
\hline CdO & 90 & 0.16 & 1.1 & 0.014 \\
CdO:Co-In-S1 & 232.9 & 1.42 & 4.44 & 0.331 \\
CdO:Co-In-S1H & 442.7 & 93.23 & 401.7 & 41.3 \\
CdO:Co-In-S2 & 307.2 & 20.1 & 65.5 & 6.2 \\
\hline
\end{tabular}




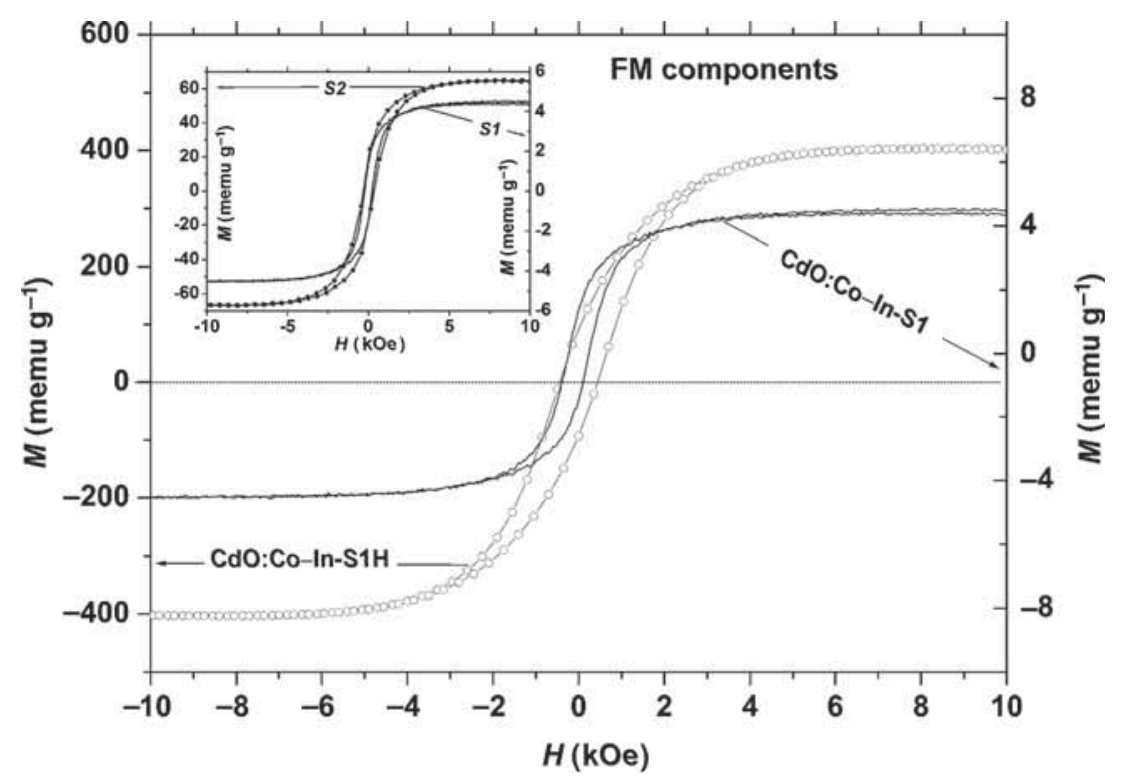

Figure 6. Comparison plot for the effect of hydrogenation on the hysteresis loop of $\mathrm{S} 1$. The inset shows a comparison plot for the effect of Co doping level on the hysteresis loops of $\mathrm{S} 1$ and $\mathrm{S} 2$ samples.

properties, more than that with S1 by increasing the dopant cobalt ions concentration; for example the saturation magnetisation was enhanced $\sim 14$ times with increasing of dopant Co ions concentration while it was enhanced $\sim 90$ times by hydrogenation means. This reflects the essential importance of the electronic medium necessary for the $S . S$ interaction. Figure 6 shows a schematic comparison between the enhancements of the hysteresis loop (FM component) by the above-mentioned two means.

It becomes known that the hydrogenation induces some structural and electronic variations, which control the properties of the electronic medium in host CdO crystal responsible for the $\boldsymbol{S . S}$ interaction and, thus, boosts the generated FM. The structural study reveals the interaction between hydrogen atoms and structural oxygen in $\mathrm{Cd}$ oxide lattice and, thus, creates oxygen vacancies $\left(\mathrm{V}_{\mathrm{O}}\right)$ with liberation of free $\mathrm{Cd}$ ions of different amounts depending on hydrogenation conditions (time and temperature). The O-vacancies supply/enhance magnetic medium for $\boldsymbol{S}$. $\boldsymbol{S}$ interaction and, thus, generate/boost FM. Therefore, the enhancing of FM properties with hydrogenation is a direct proof that O-vacancies $\left(\mathrm{V}_{\mathrm{O}}\right)$ supply a medium for the $S . S$ interaction and thus enhance the FM properties. According to the principles of bound magnetic polaron (BMP) theory $[28,29]$, the $\mathrm{O}$-vacancies bind some electrons (polarons) through which the $S . S$ exchange interaction couples the $\mathrm{Co}-3 \mathrm{~d}^{7}$ ions, causing long-range FM ordering. The strength of the S.S exchange interaction depends on the number density of polarons and O-vacancies, which could be controlled by the doping process and details of preparation and synthesis.

Figure 5 shows that in the field region $B>3 \mathrm{kOe}$, the magnetisation $(M)$ of both S1 and S2 powders is due to superimposed saturation magnetisation $\left(M_{\mathrm{s}}\right)$ of the FM contribution and PM contribution, i.e., the variation in $M(H)$ is due to PM contribution. Therefore, the slope of the straight line for $H>3$ kOe gives the PM contribution susceptibility:

$$
M(H)=M_{\mathrm{s}}+\chi_{\mathrm{m}} H, H>3 \mathrm{kOe} .
$$

The concentration of the active dopant ions interacting paramagnetically with the applied field $\left(n_{\text {ion }}\right)$ can be estimated by the Curie equation for volume susceptibility $\chi_{(\mathrm{vol})}$ in cgs units:

$$
\chi_{(\mathrm{vol})}=n_{\mathrm{ion}} \mu^{2} / 3 k_{\mathrm{B}} T,
$$

where $\mu$ is the magnetic moment per dopant ion, $k_{\mathrm{B}}$ is the Boltzmann constant and $T$ is the working temperature. From the straight-line portion (for $H>3 \mathrm{kOe}$ ) of $M-H$ graph in figure $5, \chi_{\mathrm{m}}=2.94 \times 10^{-6} \mathrm{emu}(\mathrm{g} \mathrm{Oe})^{-1}$ for S1 sample and $\chi_{\mathrm{m}}=9.21 \times 10^{-6} \mathrm{emu}(\mathrm{g} \mathrm{Oe})^{-1}$ for S2 sample. Using $\mu_{(\mathrm{Co})}=4.2 \mu_{\mathrm{B}}$, the relative concentration of magnetically active $\mathrm{Co}^{2+}$ ions is $\sim 50 \%$ for $\mathrm{S} 1$ and $\sim 53 \%$ for $\mathrm{S} 2$. In general, not all dopant $\mathrm{Co}^{2+}$ ions participate in the magnetic responses. This might be due to the medium bonds, constrains, distribution or location of ions [30,31].

\section{Conclusions}

Nanoparticle powders of solid solution $\mathrm{Cd}_{1-x-y} \operatorname{In}_{y} \mathrm{Co}_{x} \mathrm{O}$ ( $\mathrm{x}=0,0.10$ and $0.40, y=0.03$ ) have been synthesised by the solvothermal method through cadmium chloride $\left(\mathrm{CdCl}_{2}\right.$. $\mathrm{H}_{2} \mathrm{O}$ ) precursor route. The structural study showed that the precursor $\mathrm{CdCl}_{2} \cdot \mathrm{H}_{2} \mathrm{O}$ has an orthorhombic (Pnam) structure. The incorporated $\mathrm{Co} / \mathrm{In}$ ions were totally doped in the final product $\mathrm{CdO}$ lattice. The investigation confirms that the 
hydrogenation of $\mathrm{Co}-\mathrm{In}$-codoped $\mathrm{CdO}$ solid solution sample liberates $\mathrm{Cd}$ metal and induces oxygen vacancies, which boosts the FM properties of the sample. Such a phenomenon cannot happen without the presence of transition metal cobalt playing a role of catalyst. Therefore, as a general important conclusion, hydrogenation of TM-doped $\mathrm{CdO}$ would produce a nanocomposite crystal consisting of $\mathrm{Cd}$ nanoparticles immersed in TM-doped $\mathrm{CdO}$ crystalline solid medium.

The optical study showed red-shift of host $\mathrm{CdO}$ bandgap due to $\mathrm{Co} / \mathrm{In}$ doping and blue-shift with hydrogenation, which is related to a strong structural change. The magnetic investigations show that doping of $\mathrm{CdO}$ with $\mathrm{Co}^{2+} / \mathrm{In}^{3+}$ ions creates RT-FM, which strongly boosted with hydrogenation, so that $H_{\mathrm{c}}$ enhanced $\sim 2$ times, $B_{\mathrm{r}} \sim 65$ times and $B_{\text {sat }} \sim 90$ times. In general, the results demonstrate the significant importance of the electronic medium through which $\boldsymbol{S} . \boldsymbol{S}$ interaction takes place. The results of the present work demonstrate a potential candidate medium consisting of free $\mathrm{Cd}$ nanoparticles immersed in $\mathrm{Co}-\mathrm{In}$-codoped $\mathrm{CdO}$ for future DMS applications.

\section{References}

[1] Zhao Z, Morel D L and Ferekides C S 2002 Thin Solid Films 413203

[2] Dakhel A A 2012 J. Electr. Mater. 412405

[3] Shaohua Sun, Ping Wu and Pengfei Xing 2012 J. Magn. Magn. Mater. 3242932

[4] Kohan A F, Ceder G, Morgan D and Van de Walle C G 2000 Phys. Rev. B 6115027

[5] Choudhury B, Choudhurym A, Maidul Islam A K M, Alagarsmy P and Mukhherjee M 2011 J. Magn. Magn. Mater. 323440

[6] Lancashire R J 2015 Department of Chemistry, University of West Indies, Jamaica Web: http://wwwchem.uwimona.edu. $\mathrm{jm} /$ spectra/MagMom.html

[7] Shannon R D 1976 Acta Crystallogr. A 32751

[8] Ahmad Tokeer and Khatoon Sarvari 2015 J. Mater. Res. 30 1611

[9] Khatton S, Coolahan K, Lofland E S and Ahmed T 2013 J. Am. Cer. Soc. 962544
[10] Dakhel A A and Bououdina M 2014 J. Supercond. Nov. Magn. 272507

[11] Ahmad T, Khatoon S, Lofland S E and Thakur G S 2014 Mater. Sci. Semicond. Proc. 17207

[12] Gandhi V, Ganesan R, Syedahamed H H A and Thaiyan M 2014 J. Phys. Chem. C 1189715

[13] Srivastava O K and Secco E A 1967 Canadian J. Chem. 45 1375

[14] Technisch Physische Dienst 1975 Delft, Netherlands: ICDD Grant-in-Aid JCPDS File No. 05-0640

[15] Edwards D A, Wallace W E and Craig R S 1952 J. Am. Chem. Soc. 745256

[16] Yang J, Song W H, Zhang R L, Ma Y Q, Zhao B C, Sheng Z G et al 2004 Solid State Commun. 131393

[17] Dakhel A A, El-Hilo M and Bououdina M 2014 Adv. Powder Technol. 251839

[18] Lewis E A, Le D, Murphy C J, Jewell A D, Mattewra M F G, Liriano M L et al 2012 J. Phys. Chem. C 11625868

[19] Pozzo M and Alfe D 2009 Int. J. Hydrogen Energy 34 1922

[20] Schlapbach L 1980 J. Phys. F: Met. Phys. 102477

[21] Morales A E, Mora E S and Pal U 2007 Rev. Mexicana Fis. S 5318

[22] Sahin B, Bayansal F, Yuksel M and Cetinkara H A 2014 Mater. Sci. Semicond. Proc. 18135

[23] Burstein E 1954 Phys. Rev. 93632

[24] Chandiramouli R and Jeyaprakash B G 2013 Solid State Sci. 16102

[25] Bououdina M, Dakhel A A, El-Hilo M, Anjum D H, Kanoun M B and Goumri-Said S 2015 RSC Adv. 533233

[26] Seo S Y, Kwak C H, Kim S H, Park S H, Lee I J and Han S W 2012 J. Cryst. Growth 34656

[27] Raghavan V 2004 Materials science and engineering: a first course 5th edn (New Delhi: Prentic-Hall of India Private Limited), p 406

[28] Kaminski A and Sarma S D 2002 Phys. Rev. Lett. 88 247

[29] Wolff P A, Bhatt R N and Durst A C 1996 J. Appl. Phys. 7951

[30] Cheng Shun-Jen 2005 Phys. Rev. B 72235332

[31] Tolea F, Grecu M N, Kuncser V, Constantinescu S Gr and Ghica D 2015 Appl. Phys. Lett. 106142404 